\title{
TWO MORE DISK GALAXIES WITH GLOBAL GAS COUNTERROTATION*
}

\author{
O. K. SiL'Chenko ${ }^{1,2}$, A. V. Moiseev ${ }^{3}$, and V. L. AfanasieV ${ }^{3}$ \\ ${ }^{1}$ Sternberg Astronomical Institute, Moscow, 119991, Russia; olga@sai.msu.su \\ 2 Isaac Newton Institute of Chile, Moscow Branch, Russia \\ ${ }^{3}$ Special Astrophysical Observatory, Nizhnij Arkhyz, 369167, Russia; moisav@sao.ru, vafan@sao.ru \\ Received 2008 October 10; accepted 2009 January 1; published 2009 March 25
}

\begin{abstract}
We report a discovery of extended counterrotating gaseous disks in early-type disk galaxies NGC 2551 and NGC 5631. To find them, we have undertaken complex spectral observations including integral-field spectroscopy for the central parts of the galaxies and long-slit deep spectroscopy to probe the external parts. The line-of-sight velocity fields have been constructed and compared to the photometric structure of the galaxies. As a result, we have revealed full-size counterrotating gaseous disks, the one coplanar to the stellar disk in NGC 2551 and the other inclined to the main stellar disk in NGC 5631. We suggest that we observe the early stages of minor-merger events which may be two different stages of the process of lenticular galaxy formation in rather sparse environments.
\end{abstract}

Key words: galaxies: evolution - galaxies: kinematics and dynamics

Online-only material: color figures

\section{INTRODUCTION}

In principle, a significant role of mergers implied by the hierarchical paradigm for the galaxy evolution must result in frequent visible misalignments of rotation momentum between various stellar and gaseous galactic subsystems. This is especially true for noncluster lenticular galaxies whose origin should be probably due to minor-merger events.

However, findings of extended counterrotating gaseous disks are still rare. In the Sa-galaxy NGC 3626 (Ciri et al. 1995; Garcia-Burillo et al. 1998), in the S0 NGC 4546 (Bettoni et al. 1991), and in the Sb-galaxy NGC 7742 (de Zeeuw et al. 2002; Sil'chenko \& Moiseev 2006), all the gas counterrotates the stars. In the Sa-galaxies NGC 3593 (Bertola et al. 1996; Corsini et al. 1998; Garcia-Burillo et al. 2000), NGC 7217 (Merrifield \& Kuijken 1994; Sil'chenko \& Afanasiev 2000), NGC 5719 (Vergani et al. 2007), and NGC 4138 (Jore et al. 1996; Thakar et al. 1997), the counterrotating gas is already partly processed into stars, so these galaxies have two stellar counterrotating disks one of which corotates the gas. In NGC 4550 one can already see the full-size counterrotating stellar disk whereas the counterrotating gas is mainly exhausted (Rubin et al. 1992; Rix et al. 1992; Afanasiev \& Sil'chenko 2002; Emsellem et al. 2004; Sarzi et al. 2006); a similar situation may be suspected in NGC 7331 (Prada et al. 1996; Sil'chenko 1999). In the Sabgalaxy NGC 4826 (Rubin 1994; Rix et al. 1995; Braun et al. 1992, 1994) and in the S0 NGC 1596 (Chung et al. 2006), the outer gas counterrotates the inner parts of the galaxies, certainly being accreted quite recently. These few examples include almost all known extended counterrotating subsystems. Statistical estimates by Kannappan \& Fabricant (2001) and Pizzella et al. (2004) put an upper limit of 8\%-12\% of all spiral, $\mathrm{S} 0 / \mathrm{a}-\mathrm{Scd}$, galaxies to possess such structures. For S0 galaxies the appearance of counterrotating gas may be more frequent, Kuijken \& Merrifield (1996) give the estimate of $24 \pm 8 \%$; it can be consistent with the idea of S0 galaxy (trans-)formation from

\footnotetext{
* Based on the observations collected with the $6 \mathrm{~m}$ telescope (BTA) at the Special Astrophysical Observatory (SAO) of the Russian Academy of Sciences (RAS).
}

a spiral by minor merger: in such an event some external gas with decoupled momentum must be accreted. But in S0 galaxies the counterrotating gas is observed to be mostly confined to the very central part of the galaxies as it can be seen in the sample by Bertola et al. (1992); the extended counterrotating gaseous disks are rare in S0s as extended gaseous disks in general.

Another related phenomenon is inner gaseous polar disks in disk galaxies (Corsini et al. 2003). We found them as well in S0 galaxies with generally small amount of gas (Afanasiev \& Sil'chenko 2000; Sil'chenko \& Afanasiev 2004) as in spiral galaxies with normal extended H I disks-NGC 2841 (Sil'chenko et al. 1997), NGC 7217 (Sil'chenko \& Afanasiev 2000), and NGC 7468 (Shalyapina et al. 2004). For the inner polar disk origin the most popular hypothesis is also external gas accretion; however in some cases dynamical simulations predict strongly inclined circumnuclear gaseous disks produced by secular evolution processes. The simulations by Friedli $\&$ Benz (1993) of the isolated stellar-gaseous disk evolution gave such "polar disks" as a result of gas redistribution in the global disk of a galaxy, if initially all the gas in the disk counterrotated the stars. Interestingly, the old question, which came first, the hen or the egg, still exists concerning this problem. Van Albada et al. (1982) obtained a similar configuration, the inner polar disk plus the outer counterrotating gas, starting from a single inner polar disk: in a tumbling triaxial potential the outer parts of the gaseous polar disk warped in their model so that the outer gas counterrotated the stars almost in the main symmetry plane. By paying attention to the outer extension of the inner gaseous polar disks found by us, we have revealed indeed some cases of the configuration required, the inner polar disk plus the more outer counterrotation: these are the lenticular galaxies NGC 7280 and NGC 7332 (Sil'chenko 2005), IC 1548 (Sil'chenko \& Afanasiev 2008), and the late-type spiral galaxy NGC 7625 (Moiseev 2008).

An exceptionality of the extended counterrotating disks means that there must exist some additional conditions for a disk galaxy to retain large masses of accreted counterrotating gas; perhaps, it may be a very low rate of the acquisition process (Thakar \& Ryden 1996), or the absence of a large amount of initial, "own" galactic gas in the recipient galaxy (Pizzella 
Table 1

Global Parameters of the Galaxies

\begin{tabular}{lcc}
\hline \hline NGC & 2551 & 5631 \\
\hline Type $\left(\mathrm{NED}^{\mathrm{a}}\right)$ & $\mathrm{SA}(\mathrm{s}) 0 / \mathrm{a}$ & $\mathrm{SA}(\mathrm{s}) 0^{0}$ \\
$R_{25}, \mathrm{kpc}^{\mathrm{L}}\left(\mathrm{LEDA}^{\mathrm{b}}\right)$ & 8.5 & 9.3 \\
$B_{T}^{0}\left(\mathrm{RC3}^{\mathrm{c}}\right)$ & 12.78 & 12.35 \\
$M_{B}(\mathrm{LEDA})$ & -20.0 & -20.2 \\
$(B-V)_{T}^{0}(\mathrm{RC} 3)$ & 0.92 & 0.90 \\
$V_{r}(\mathrm{NED}), \mathrm{km} \mathrm{s}^{-1}$ & 2344 & 1979 \\
Distance, Mpc (LEDA) & 37.1 & 32.1 \\
Inclination $(\mathrm{LEDA})$ & $50^{\circ}$ & $21^{\circ}$ \\
P.A.phot $(\mathrm{LEDA})$ & $52^{\circ}$ & $\ldots$ \\
$V_{\text {rot }} \sin i, \mathrm{~km} \mathrm{~s}^{-1}(\mathrm{LEDA}, \mathrm{HI})$ & $83.6 \pm 5.1$ & $165.4 \pm 14.3$ \\
$M_{\mathrm{HI}}{ }^{\mathrm{d}}, 10^{9} M_{\odot}$ & 1.4 & 1.4 \\
\hline
\end{tabular}

Notes.

${ }^{a}$ NASA/IPAC Extragalactic Database.

${ }^{\mathrm{b}}$ Lyon-Meudon Extragalactic Database.

${ }^{c}$ Third Reference Catalogue of Bright Galaxies.

${ }^{\mathrm{d}}$ Bettoni et al. (2003).

et al. 2004). Every new disk galaxy with a globally counterrotating gas component may in principle help to determine these conditions. In this paper, we report the discovery of two more extended counterrotating gaseous disks in early-type disk galaxies. We present the results of the kinematical study of the nearby S0 galaxies NGC 2551 and NGC 5631. NGC 2551 and NGC 5631 galaxies considered in this paper are early-type disk galaxies of intermediate luminosity. Both belong to spiral-dominated groups (Giuricin et al. 2000), and both have a substantial amount of rotating neutral hydrogen with unknown sense of rotation, according to single-dish radio observations at $21 \mathrm{~cm}$ (Balkowski \& Chamaraux 1983; Theureau et al. 1998). Their main parameters retrieved in databases and from literature are presented in the Table 1. The layout of the paper as follows. In Section 2 we describe our observations, our data reduction, and some additional information. In Section 3, we present the counterrotating gaseous disk in NGC 2551, and in Section 4, the complex kinematics including inclined counterrotating stellar-gaseous disk in NGC 5631. Section 5 contains some discussion on the origin and the possible fate of the counterrotating gas in these two galaxies.

\section{OBSERVATIONS AND DATA REDUCTION}

To study the rotation of stars and ionized gas, we use spectral data obtained for NGC 2551 and NGC 5631 with three different spectrographs.

The Multi-Pupil Fiber Spectrograph (MPFS) of the Russian $6 \mathrm{~m}$ telescope (Afanasiev et al. 2001) is an integral-field unit constructed by following the fiber-lens principle; due to this feature it allows to obtain panoramic spectral data over a wide spectral range (in our case, over $1500 \AA$ with the spectral resolution of $3 \AA$ ). The field of view (FOV) is $16^{\prime \prime} \times 16^{\prime \prime}$, with the sampling of $1^{\prime \prime}$ per microlens. We observed NGC 2551 and NGC 5631 in the green spectral range containing a lot of absorption lines and calculated the line-of-sight (LOS) stellar velocities by cross-correlating continuum-subtracted and logarithmically binned galactic spectra with the similarly prepaired spectra of the twilight (the Sun spectrum, of G2 spectral type) and of G- and K-giant stars observed the same nights as the galaxiesHD 19476 (K0III) for NGC 2551 and HD 135722 (G8III), and HD 167042 (K1III) for NGC 5631. For NGC 2551, also the red spectral range has been exposed to make Gauss fitting of the
[N II] 26583 emission line and to estimate the LOS velocities of the ionized gas. The statistical accuracy of one-element LOS velocity and velocity dispersion estimates with the MPFS data is about $10 \mathrm{~km} \mathrm{~s}^{-1}$.

In 2007, the galaxy NGC 5631 was also observed with another integral-field spectrograph, the SAURON of the William Herschel Telescope at La Palma (Bacon et al. 2001). We have retrieved these data from the open ING archive of the Cambridge Astronomical Data Center and have reduced them in our manner calculating the stellar LOS velocities by cross-correlation with the spectrum of a star observed the same night (HD 72184, $\mathrm{K} 2 \mathrm{III}$, this time) and by calculating the gas LOS velocities by measuring the barycenter positions of the [O III] $\lambda 5007$ emission line in the continuum-subtracted spectra. The FOV of the SAURON is $33^{\prime \prime} \times 41^{\prime \prime}$, with the sampling of $00^{\prime} 94$; the spectral resolution is $4 \AA$ and the spectral range is narrow, 4800-5350 $\AA$, because it is a TIGER-mode integral-field spectrograph.

After discovering central gas counterrotation in both galaxies with the integral-field spectroscopic data, we wanted to know the full extension of the counterrotating gas systems. To check that, we have observed the galaxies with the spectrograph SCORPIO of the $6 \mathrm{~m}$ telescope in the long-slit mode (Afanasiev \& Moiseev 2005). The slit, whose length is about 6 arcmin, has been aligned with the kinematical major axis of the central LOS velocity fields. The red spectral range, 6100-7100 $\AA$, with a $2.5 \AA$ spectral resolution, has been exposed to measure first of all the LOS velocities of the strongest emission line in the optical spectral range, $[\mathrm{N}$ II] $\lambda 6583$, which is free of the underlying absorption contamination unlike the $\mathrm{H} \alpha$. However, the LOS velocity and stellar velocity dispersion profiles for the stellar components have also been estimated by crosscorrelating galactic spectra binned along the slit with a template star spectrum from the library MILES (Sanchez-Blazquez et al. 2006). Through the library we chose spectra of the stars HD 48433 (K1III) and HD 10380 (K3III), which provided the largest amplitudes of the cross-correlation function with the spectra of NGC 2551 and NGC 5631, respectively. We measured the errors of the stellar velocity and velocity dispersion by using the formulae from the classical paper by Tonry \& Davis (1979). The uncertainties of the ionized-gas kinematical parameters were estimated by Monte Carlo simulations of artificial spectra with the noise distribution similar to that of the original data.

The log of observations is given in Table 2 .

To analyze the morphological structure of the galaxies, we have also used surface photometric data obtained by reducing the digital images from the Hubble Space Telescope (HST) Archive (NGC 2551, ACS/F625W) and from the Sloan Digital Sky Survey (SDSS)/DR6 (Adelman-McCarthy et al. 2008) data collection (NGC 5631, $g^{\prime}, r^{\prime}, i^{\prime}, z$-filters).

\section{COUNTERROTATING STARFORMING GASEOUS DISK IN NGC 2551}

Figure 1 presents the LOS velocity fields for the stellar and gaseous components in the center of NGC 2551 which we have obtained with the MPFS. The seeing quality has been much worse during the red-range MPFS exposure, so the visible gas rotation seems to be slower than that of the stars; it is an effect of smearing the steep velocity gradient by poor spatial resolution. In general, Figure 1 is intended only to demonstrate gas counterrotation with respect to the stars in the center of the galaxy. The directions of the kinematical major axis which is defined as a direction of the maximum 
Table 2

The Observational Log

\begin{tabular}{|c|c|c|c|c|c|c|c|}
\hline Date & Galaxy & Exposure & Spectrograph & Field of View & P.A.(top) & Spectral Range & Seeing \\
\hline 2007 Aug 20 & NGC 2551 & $60 \mathrm{~min}$ & BTA/MPFS & $16^{\prime \prime} \times 16^{\prime \prime}$ & $179^{\circ}$ & $4200-5600 \AA$ & $1^{\prime \prime} .5$ \\
\hline 2008 Mar 7 & NGC 2551 & $56 \mathrm{~min}$ & BTA/MPFS & $16^{\prime \prime} \times 16^{\prime \prime}$ & $179^{\circ}$ & $5800-7200 \AA$ & $3 . .0$ \\
\hline 2008 Jan 15 & NGC 2551 & $40 \mathrm{~min}$ & BTA/SCORPIO & $1^{\prime \prime} \times 360^{\prime \prime}$ & $55^{\circ}$ & $6100-7100 \AA$ & $1 " .5$ \\
\hline 2007 Aug 17 & NGC 5631 & $80 \mathrm{~min}$ & BTA/MPFS & $16^{\prime \prime} \times 16^{\prime \prime}$ & $292^{\circ}$ & $4200-5600 \AA$ & 2.0 \\
\hline 2007 Apr 24 & NGC 5631 & $60 \mathrm{~min}$ & WHT/SAURON & $33^{\prime \prime} \times 41^{\prime \prime}$ & $307^{\circ}$ & $4800-5350 \AA$ & $1^{\prime \prime} .5$ \\
\hline 2008 Apr 6 & NGC 5631 & $49 \mathrm{~min}$ & BTA/SCORPIO & $1^{\prime \prime} \times 360^{\prime \prime}$ & $115^{\circ}$ & $6100-7100 \AA$ & $3 . .5$ \\
\hline 2008 May 13 & NGC 5631 & $26 \mathrm{~min}$ & BTA/SCORPIO & $1^{\prime \prime} \times 360^{\prime \prime}$ & $140^{\circ}$ & $6100-7100 \AA$ & $2^{\prime \prime} .5$ \\
\hline
\end{tabular}
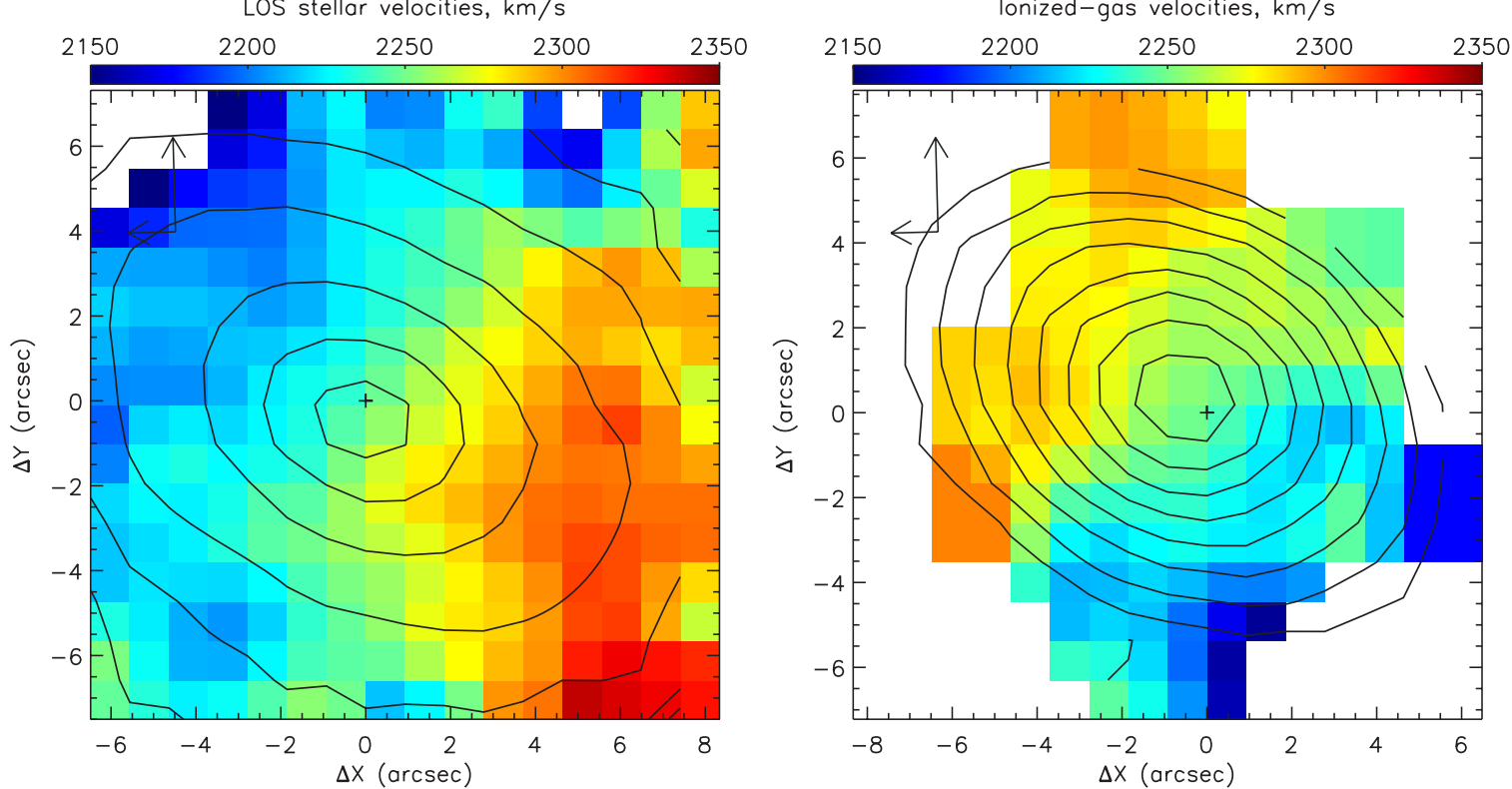

stellar velocity dispersion, $\mathrm{km} / \mathrm{s}$

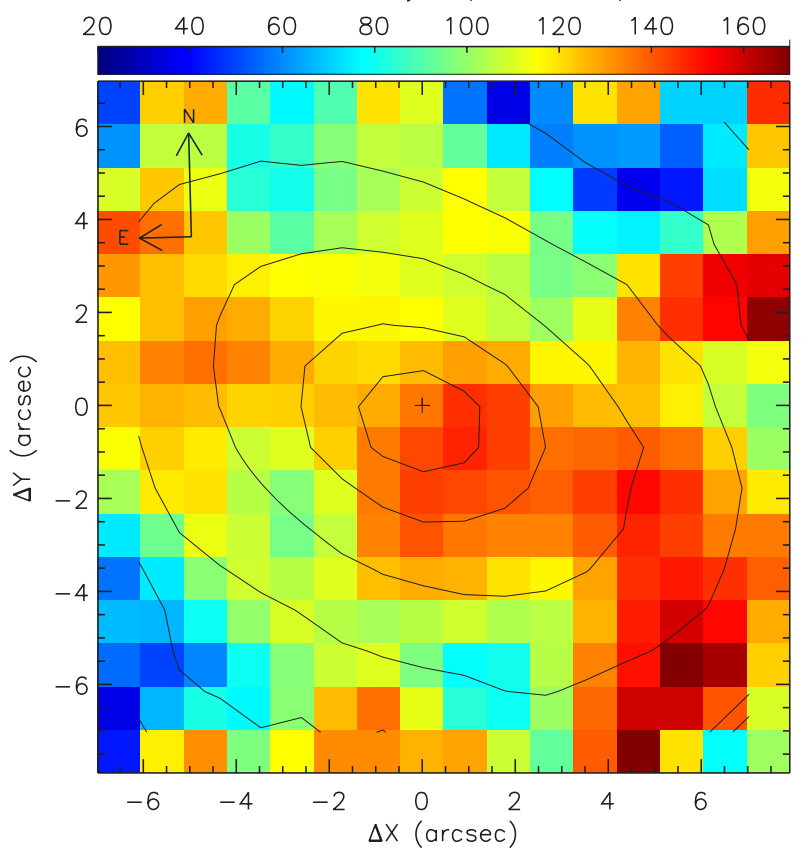

Figure 1. LOS velocity fields of stars (upper left) and ionized gas (upper right) in the central part of NGC 2551 obtained with the MPFS, as well as the stellar velocity dispersion field (bottom). The isophotes of the continuum at $\lambda 5000 \AA$ (left and bottom) and at $\lambda 6500 \AA$ (right) are superposed.

(A color version of this figure is available in the online journal.)

LOS velocity gradient are P.A. $=236^{\circ} \pm 2^{\circ}$ for the stellar component and P.A. $=36^{\circ} \pm 10^{\circ}$ for the ionized gas. The former value coincides with the photometrical major axis direction, P.A.phot $=54^{\circ} \pm 1^{\circ}$ at $R=1^{\prime \prime}-3^{\prime \prime}$ according to the HST data, 


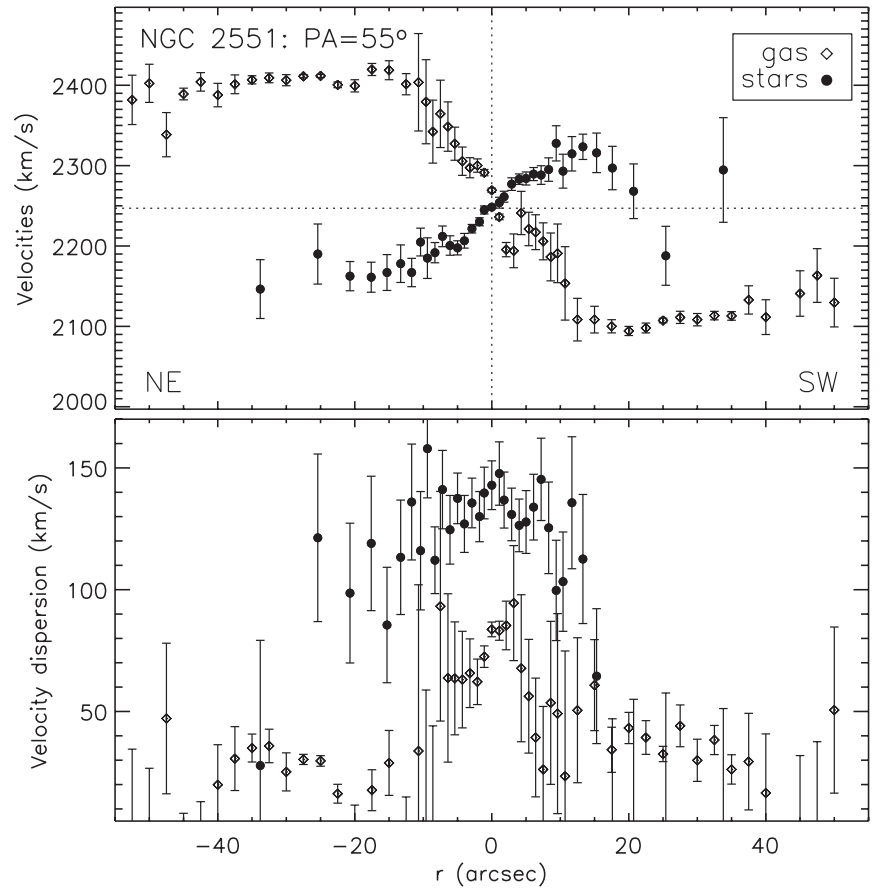

Figure 2. LOS velocity and velocity dispersion profiles of stars and ionized gas obtained for NGC 2551 with the SCORPIO at P.A. $=55^{\circ}$.

implying an axisymmetric character of the galaxy kinematics and structure. Indeed, NGC 2551 is known to be unbarred, and its photometric major axis direction, P.A. $\approx 52^{\circ}-55^{\circ}$, is constant along the full radial extension (Mizuno \& Hamajima 1987). The fact that the isophote ellipticity reaches its maximum, $1-b / a=0.38$, already at $R \approx 6^{\prime \prime}\left(0.1 R_{25}\right)$ implies that the galaxy is disk-dominated. This conclusion is confirmed by the major-axis surface brightness profile decomposition by Baggett et al. (1998): according to their model, the regular exponential disk dominates in the brightness profile of NGC 2551 starting from $R \approx 8^{\prime \prime}$.

The next question, which arose after the gas counterrotation was found with the MPFS, was whether to deal with the central decoupled gas subsystem, or the counterrotating gas extended over the whole galaxy. The SCORPIO gas and stellar LOS velocity profiles obtained at P.A. $=55^{\circ}$ (Figure 2 ) demonstrate persistence of the gas-star counterrotation up to $R=35^{\prime \prime}$ at least. The gas rotation curve is rather flat and extended to $R=$ $50^{\prime \prime} \approx R_{25}$. The projected gas rotation velocity, $150 \mathrm{~km} \mathrm{~s}^{-1}$, exceeds even the aperture H I value from Balkowski \& Chamaraux (1983) (Table 1) so P.A. $=55^{\circ}$ may well be the global disk line-of-nodes direction. In general, the data favor coplanar stellar and gaseous disks in NGC 2551, though rotating in opposite senses.

\section{COUNTERROTATING GAS IN NGC 5631}

Figure 3 presents the stellar and gas LOS velocity fields for the center of NGC 5631 constructing by using the data of the MPFS and the SAURON; the LOS velocities of the ionized gas have been calculated by measuring the barycenter positions of the emission line [O III] $\lambda 5007$. The stars and the ionized gas counterrotate over the whole FOV of the MPFS, and there is a hint of the reverse rotation for the stars at the edges of the SAURON FOV. The orientations of the kinematical major axes within $5^{\prime \prime}$ from the center, P.A. $=118^{\circ} \pm 4^{\circ}$ for the stars and
P.A. $\approx 300^{\circ}$ for the ionized gas, are consistent with each other and with the photometric major axis orientation in the central part of the galaxy, P.A.phot $=126^{\circ} \pm 2^{\circ}$, implying coplanar axisymmetric rotation.

The long-slit cross sections made with the SCORPIO have shown that the gaseous disk which rotation we observe in the center of NGC 5631 is rather extended: we see the measurable emission lines up to $R \approx 35^{\prime \prime}\left(0.7 R_{25}\right)$ (Figure 4$)$. The gas excitation is shocklike over the full extension of the visible emission: the $[\mathrm{N} \mathrm{II}] \lambda 6583$ emission line is everywhere stronger than the $\mathrm{H} \alpha$ one. The maximum projected rotation velocity reaches about $170 \mathrm{~km} \mathrm{~s}^{-1}$, being again consistent with the integrated H I data (Theureau et al. 1998). The stellar component counterrotates the ionized gas up to $R=10^{\prime \prime}-15^{\prime \prime}$. At $R=$ $15^{\prime \prime}$ the projected rotation velocity of the stars falls to zero. Interestingly, the same character of the stellar LOS velocity profile was found by Simien \& Prugniel (2002) who obtained a long-slit cross section at P.A. $=135^{\circ}$ : the maximum velocity, $v_{\text {rot }} \sin i=51 \pm 6 \mathrm{~km} \mathrm{~s}^{-1}$, was reached at $R \approx 10^{\prime \prime}$, then the velocity curve fell, passed through zero at $R \approx 20^{\prime \prime}$, and reversed its sense at $R>20^{\prime \prime}$. The stellar velocity dispersion profile at P.A. $=115^{\circ}$ demonstrates some rise at $R \sim 10^{\prime \prime}$. This feature becomes understandable if we look directly at the line-of-sight velocity distribution (LOSVD) shape (Figure 5). At $R=-12^{\prime \prime}$ and at $R=+14^{\prime \prime}$ the LOSVD becomes asymmetric with the hint on two peaks and remains two-peaked up to the limit of our measurements, $R \approx 30^{\prime \prime}$. This fact results in the visible increase of the stellar velocity dispersion and in the visible fall of the projected rotation velocity to zero: two counterrotating stellar components compensate each other being approximated by a single Gaussian LOSVD.

The isophote behavior is not so simple in NGC 5631 as in NGC 2551: though the galaxy is unbarred, the isophotes of the central part, within $R \sim 40^{\prime \prime}$ from the center, are more elliptical than those of the outer disk, and their major axis is turning perhaps between $R \approx 3^{\prime \prime}$ and $R \approx 25^{\prime \prime}$ from P.A. $\approx 120^{\circ}$ to P.A. $=138^{\circ}$ (Figure 6$)$. The shape of the brightness profiles, which we have derived from the SDSS data by averaging the counts per arcsec over the ellipses with the parameters found by the isophote analysis, allows us to divide the whole galaxy into three main components (Figure 7). The outer exponential stellar disk dominating at $R>50^{\prime \prime}$ is seen face-on: the ellipticity of the isophotes is less than 0.05 , and the orientation of the major axis cannot be determined. The inner exponential stellar component (inner disk?) is clearly seen in the radius range of $12^{\prime \prime}-35^{\prime \prime}$; the orientation of the isophote major axis is P.A. $=137^{\circ}-138^{\circ}$, and the isophote ellipticity after subtracting the outer disk stays at $0.17-0.18$ implying an inclination of $\sim 35^{\circ}$ under the assumption of a rather thin disk. The surface brightness profile of the bulge, $R<8^{\prime \prime}$, can be approximated by Sersic law for $n=2$ with high accuracy; however, the isophote ellipticity and major-axis orientation within this central zone do not stay constant along the radius. The recent work by Méndez-Abreu et al. (2008) suggests the brightness profile decomposition of NGC 5631 into only two components, the Sersic bulge and the single exponential disk; but as one can see in Figure 7, their use of the shallow Two Micron All Star Survey (2MASS) photometry does not allow them to measure the outer stellar disk in NGC 5631.

The change of the stellar rotation direction takes place within the inner disk; the isophote ellipticity does not fall to zero at $R=12^{\prime \prime}-25^{\prime \prime}$, on the contrary, it stays constant at $0.17-$ 0.18 . So we conclude that the observed stellar LOS velocity behavior cannot be due to a rotation plane warp in the nearly 

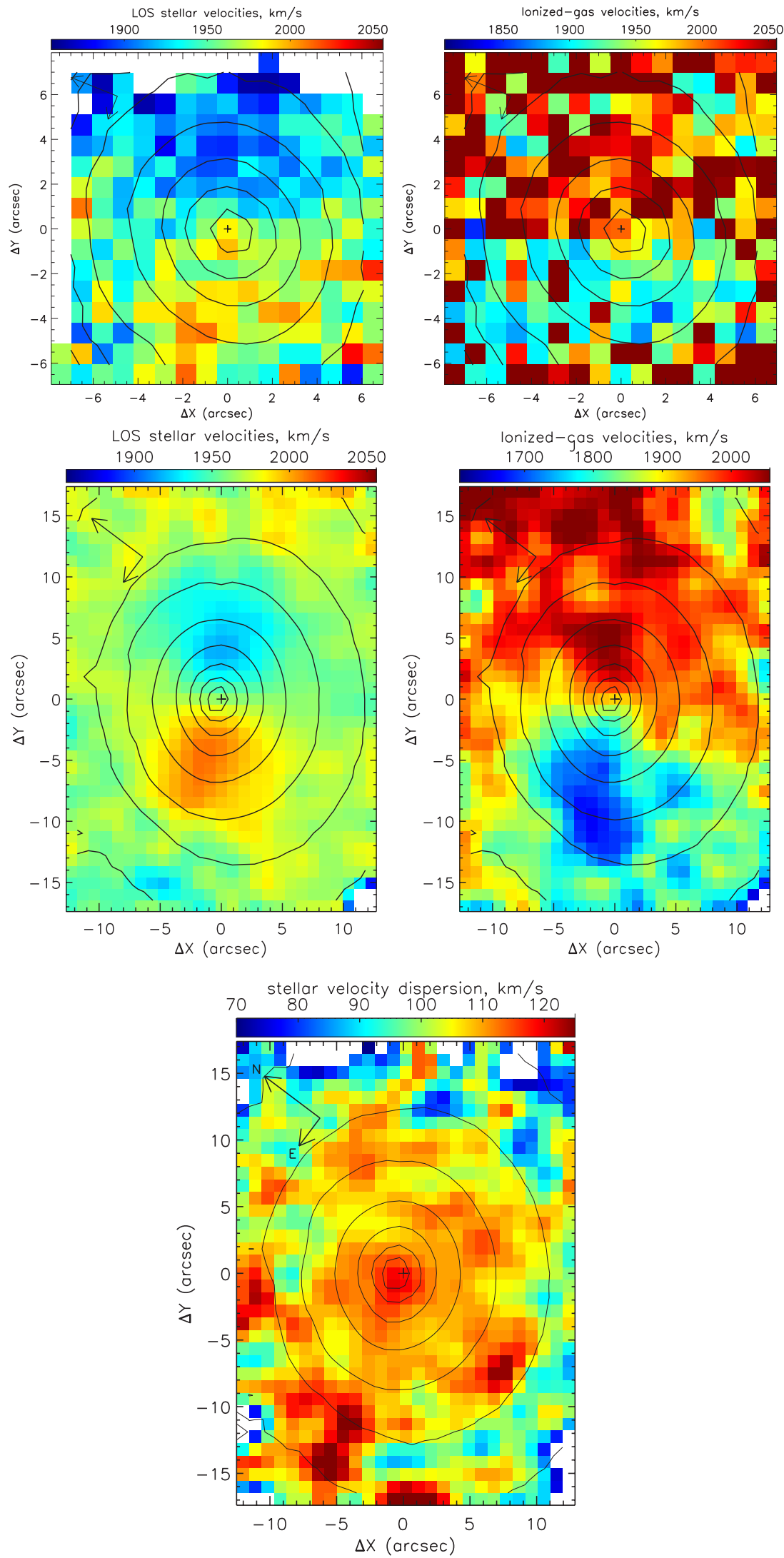

Figure 3. LOS velocity fields of stars (left column plots) and of ionized gas (right column plots) in the central part of NGC 5631 obtained with the MPFS (upper row) and with the SAURON (middle row); also the stellar velocity dispersion field obtained with the SAURON is shown at the bottom. The SAURON maps are smoothed to stress the rotation sense reverse in the outer part of the FOV. The isophotes of the green $(\lambda 5000 \AA)$ continuum are superposed. 

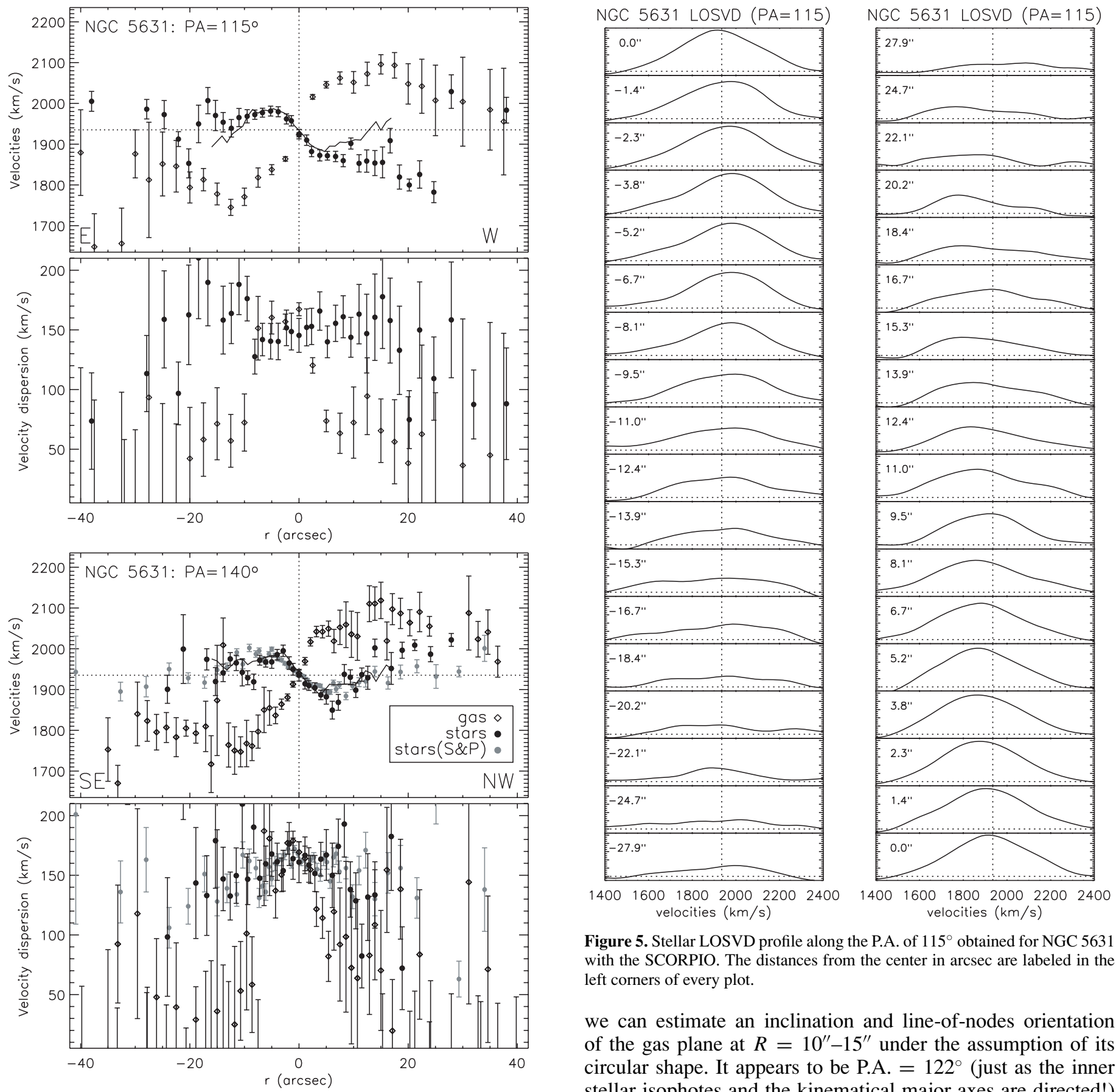

Figure 4. LOS velocity (top) and velocity dispersion (bottom) profiles of stars and ionized gas obtained for NGC 5631 with the SCORPIO with two position angles (P.A.s). By solid lines we superpose the digital-slit cross sections of the SAURON stellar LOS velocity field. Also the measurements by Simien \& Prugniel (2002) in P.A. $=135^{\circ}$ are plotted over our data for P.A. $=140^{\circ}$ for comparison.

face-on galaxy, but is indeed a manifestation of the switch of the mean stellar rotation direction inside the zone of the photometric dominance of the inner stellar disk.

Fortunately, we can say something about the gas plane orientation too. Figure 8 presents a color map of NGC 5631 that we have constructed by using the SDSS data; for the SDSS survey description, see York et al. (2000). At the radius of $R=10^{\prime \prime}-15^{\prime \prime}$ one can see a broad red (dust) ring. More exactly we see a half of the dust ring, the other half being hidden behind the bulge. Since the gas is thought to be coupled with the dust,

Figure 5. Stellar LOSVD profile along the P.A. of $115^{\circ}$ obtained for NGC 5631 with the SCORPIO. The distances from the center in arcsec are labeled in the left corners of every plot.

we can estimate an inclination and line-of-nodes orientation of the gas plane at $R=10^{\prime \prime}-15^{\prime \prime}$ under the assumption of its circular shape. It appears to be P.A. $=122^{\circ}$ (just as the inner stellar isophotes and the kinematical major axes are directed!) and $1-b / a=0.18$ - just as the stellar isophotes within the inner disk. Then the deprojected gas rotation velocity under the assumption of $i=35^{\circ}$ is around $360-390 \mathrm{~km} \mathrm{~s}^{-1}$ within the model of circular rotation that is high but not exceptional.

Some asymmetry of the stellar LOS velocity profiles in Figure 4 could then be explained if we assume that the inclined gaseous disk with the orientation parameters deduced above contains some stellar component also, and both are coupled with the inner stellar disk derived from the surface brightness profile decomposition. Then the dusty stellar disk dominating photometrically in the radius range of $R=10^{\prime \prime}-30^{\prime \prime}$ and inclined with respect to the face-on main stellar disk would completely hide the main stellar component to the north of the center; just this picture is observed in the cross section at P.A. $=140^{\circ}$. The slit at P.A. $=115^{\circ}$, projected to the west below the line of nodes of this dusty disk, catches the rotation of the main 


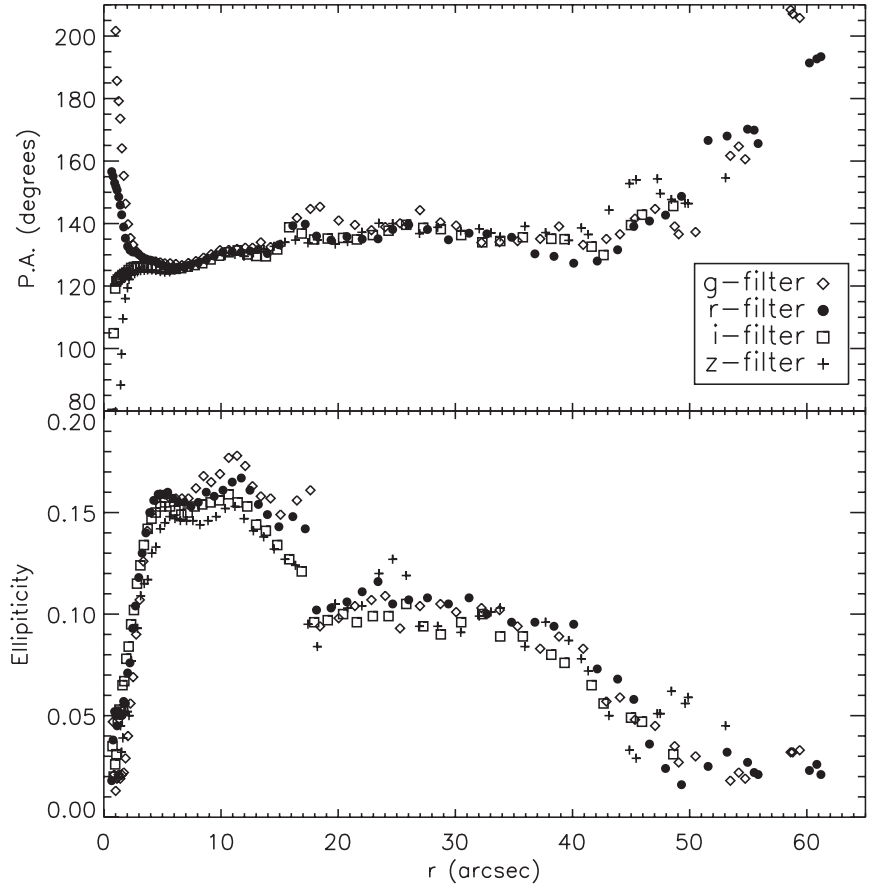

Figure 6. Radial variations of the isophote parameters found for NGC 5631 from the analysis of the SDSS photometric images

stellar component (a long western receding velocity branch, Figure 4(a)). And both cross-sections in their eastern parts demonstrate the zero mean LOS velocities and the visible stellar velocity dispersion raising up to $180 \mathrm{~km} \mathrm{~s}^{-1}$ at $R=10^{\prime \prime}-20^{\prime \prime}$, which can be treated as a superposition of two comparable stellar components counterrotating each other.

\section{DISCUSSION AND CONCLUSIONS}

Both NGC 2551 and NGC 5631 possess the extended (up to $0.7-1.0 R_{25}$, or up to $5-7 \mathrm{kpc}$ from the center) counterrotating gaseous disks. Both galaxies belong to loose groups dominated by spiral galaxies (Garcia 1993; Geller \& Huchra 1983). However neither NGC 2551 nor NGC 5631 has a close neighbor within the circle of $100 \mathrm{kpc}$ radius to provide interaction and smooth gas accretion that is suggested by Thakar \& Ryden (1996) to be the most probable mechanism of massive counterrotating disk formation. The only alternative which is available for NGC 2551 and NGC 5631 is a minor merger with a gas-rich satellite. We do not know what morphological type the galaxies NGC 2551 and NGC 5631 had before merging, and if they have had their own (corotating) gas. But in any case the accreted gas had to suffer instantaneous star formation triggered by shock compression during the merging. Perhaps, in NGC 2551 and NGC 5631 we see different stages of the same process. Ultraviolet imaging with the UIT and later on with the GALEX has revealed an extended, up to $R=40^{\prime \prime}$, star-forming disk in NGC 2551 (Marcum et al. 2001; Gil de Paz et al. 2007). An intensity ratio $\mathrm{H} \alpha /[\mathrm{N}$ II] observed by us with the SCORPIO implies an excitation by young stars up to $40^{\prime \prime}$; however, between $R=40^{\prime \prime}$ and $R=50^{\prime \prime}$ we see only one emission line, $[\mathrm{N}$ II $] \lambda 6583$, so in this ring the gas excitation may be of shock origin. In NGC 5631 there is no current star formation in the counterrotating gaseous disk, and the gas is excited by shock over the full extension of the gaseous disk. Perhaps, it is the preceding evolutionary stage with respect to NGC 2551, the accreted gas disk has not yet settled into the symmetry plane
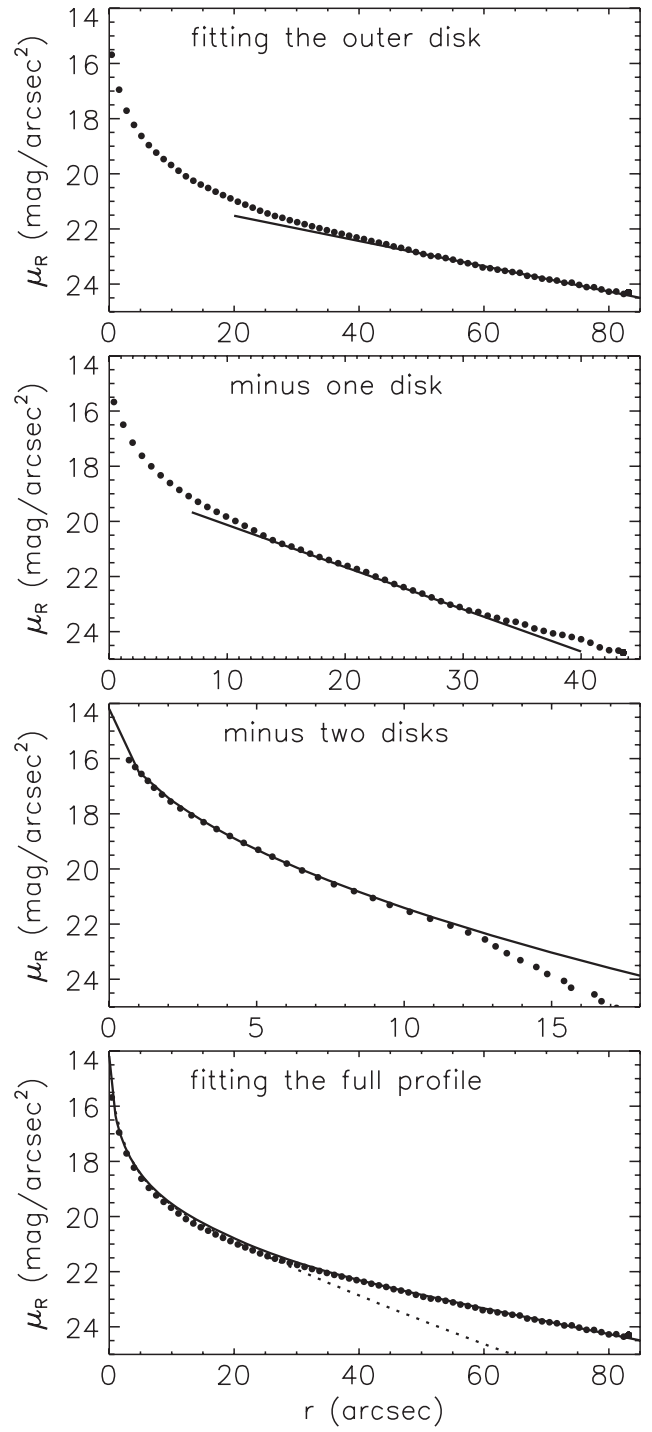

Figure 7. Surface brightness profile decomposition into three components for NGC 5631: two exponential disks with different inclinations, $i \approx 0^{\circ}$ for the outer one, $i \approx 35^{\circ}$ for the inner one, and one Sérsic bulge with $n=2$. At the last plot we compare the observed SDSS $r^{\prime}$-band brightness profile of NGC 5631 with our full model profile and with the decomposition proposed by MéndezAbreu et al. (2008) for the 2MASS J-band image of NGC 5631 (the dotted line, normalized arbitrarily).

of the main galaxy, and star formation is only going to start in the compressed inner dusty ring at $R=10^{\prime \prime}-15^{\prime \prime}$. And what may be the subsequent stage? Perhaps, it is NGC 4138 where a counterrotating extended gas is supplemented by the substantial counterrotating young stellar component (Thakar et al. 1997) - the evident consequence of the star formation in the counterrotating gaseous disk.

To give a conclusion, we summarize that by applying complex spectral methods including integral-field spectroscopy to the central parts of the galaxies and long-slit deep spectroscopy to probe the external parts, we have found two more global gas counterrotating systems in noninteracting early-type disk galaxies. In NGC 2551 two counterrotating disks, gaseous and stellar ones, may be coplanar: the orientation parameters of the optical-band image and of the UV-band, star formation related image are similar. In NGC 5631 the gaseous disk is inclined by some $35^{\circ}$ to the main stellar disk; it may also contain significant coupled stellar component. The totality of the spectral and photometric data gives evidence for the minor 

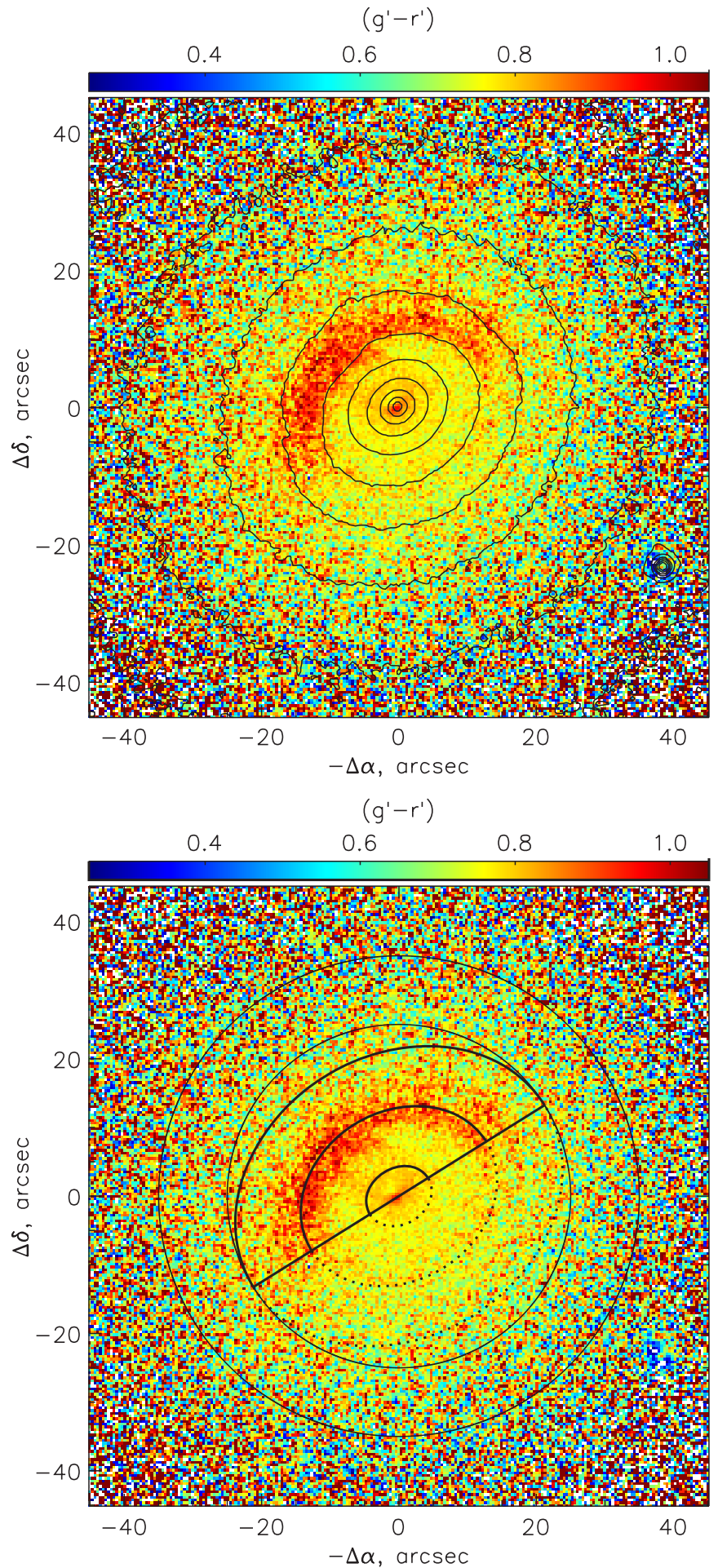

Figure 8. Color map $\left(\mathrm{g}^{\prime}-\mathrm{r}^{\prime}\right)$ of NGC 5631 from the SDSS data. The $r^{\prime}$-band isophotes (top) and the schematic view of the both disks (bottom) are overlapped onto the color map. The derived orientation of the inner inclined gaseous/dust disk is $i=35^{\circ}$, P.A. $=122^{\circ}$

(A color version of this figure is available in the online journal.)

merging as the most probable origin of the counterrotating gas in these galaxies. Perhaps, we observe two different stages of the process of lenticular galaxy formation in rather sparse group environments.

The $6 \mathrm{~m}$ telescope is operated under the financial support of the Ministry of Science and Education (registration number 01-43). Our study of the galaxies with the multitier disks, such as NGC 5631, is supported by the grant of the Russian Foundation for Basic Researches No. 07-02-00229. A.V.M. acknowledges a grant from the President of the Russian Federation (MK1310.2007.2). During the data analysis we have used the Lyon-Meudon Extragalactic Database (HYPERLEDA) supplied by the LEDA team at the CRAL-Observatoire de Lyon (France) and the NASA/IPAC Extragalactic Database (NED) which is operated by the Jet Propulsion Laboratory, California Institute of Technology, under contract with the National Aeronautics and Space Administration (NASA). This research is partly based on data obtained from the Isaac Newton Group Archive that is maintained as part of the CASU Astronomical Data Center at the Institute of Astronomy, Cambridge, on observations made with the NASA/ESA Hubble Space Telescope, obtained from the data archive at the Space Telescope Science Institute, which is operated by the Association of Universities for Research in Astronomy, Inc., under NASA contract NAS 5-26555, and on the SDSS data. Funding for the SDSS and SDSS-II has been provided by the Alfred P. Sloan Foundation, the Participating Institutions, the National Science Foundation, the U.S. Department of Energy, NASA, the Japanese Monbukagakusho, and the Max Planck Society, and the Higher Education Funding Council for England. The SDSS Web site is http://www.sdss.org/.

\section{REFERENCES}

Adelman-McCarthy, J., et al. 2008, ApJS, 175, 297

Afanasiev, V. L., Dodonov, S. N., \& Moiseev, A. V. 2001, in Stellar Dynamics: From Classic to Modern, ed. L. P. Osipkov \& I. I Nikiforov (St. Petersburg: St. Petersburg Univ. Press), 103

Afanasiev, V. L., \& Moiseev, A. V. 2005, Astron. Lett., 31, 193

Afanasiev, V. L., \& Sil'chenko, O. K. 2000, AJ, 119, 126

Afanasiev, V. L., \& Sil'chenko, O. K. 2002, AJ, 124, 706

Bacon, R., et al. 2001, MNRAS, 326, 23

Baggett, W. E., Baggett, S. M., \& Anderson, K. S. J. 1998, AJ, 116, 1626

Balkowski, C., \& Chamaraux, P. 1983, A\&AS, 51, 331

Bertola, F., Buson, L. M., \& Zeilinger, W. W. 1992, ApJ, 401, L79

Bertola, F., et al. 1996, ApJ, 458, L67

Bettoni, D., Galletta, G., \& García-Burillo, S. 2003, A\&A, 405, 5

Bettoni, D., Galletta, G., \& Oosterloo, T. 1991, MNRAS, 248, 544

Braun, R., Walterbos, R. A. M., \& Kennicutt, R. C., Jr. 1992, Nature, 360, 442

Braun, R., Walterbos, R. A. M., Kennicutt, R. C. Jr., \& Tacconi, L. J. 1994, ApJ, 420,558

Chung, A., Koribalski, B., Bureau, M., \& van Gorkom, J. H. 2006, MNRAS, 370,1565

Ciri, R., Bettoni, D., \& Galletta, G. 1995, Nature, 375, 661

Corsini, E. M., Pizzella, A., Coccato, L., \& Bertola, F. 2003, A\&A, 408, 873 Corsini, E. M., Pizzella, A., Funes, J. G., Vega Beltran, J. C., \& Bertola, F. 1998 , A\&A, 337, 80

Emsellem, E., et al. 2004, MNRAS, 352, 721

Friedli, D., \& Benz, W. 1993, A\&A, 268, 65

Garcia, A. M. 1993, A\&AS, 100, 47

García-Burillo, S., Sempere, M. J., \& Bettoni, D. 1998, ApJ, 502, 235

García-Burillo, S., Sempere, M. J., Combes, F., Hunt, L. K., \& Neri, R. 2000, A\&A, 363, 869

Geller, M. J., \& Huchra, J. P. 1983, ApJS, 52, 61

Gil de Paz, A., et al. 2007, ApJS, 173, 185

Giuricin, G., Marinoni, C., Ceriani, L., \& Pisani, A. 2000, ApJ, 543, 178

Jore, K. P., Broeils, A. H., \& Haynes, M. P. 1996, AJ, 112, 438

Kannappan, S. J., \& Fabricant, D. G. 2001, AJ, 121, 140

Kuijken, K., \& Merrifield, M. R. 1996, MNRAS, 283, 543

Marcum, P. M., et al. 2001, ApJS, 132, 129

Méndez-Abreu, J., Aguerri, J. A. L., Corsini, E. M., \& Simonneau, E. 2008, A\&A, 478, 353

Merrifield, M. R., \& Kuijken, K. 1994, ApJ, 432, 575

Mizuno, T., \& Hamajima, K. 1987, PASJ, 39, 221

Moiseev, A. V. 2008, Astrophys. Bull., 63, 201

Pizzella, A., Corsini, E. M., Vega Beltrán, J. C., \& Bertola, F. 2004, A\&A, 424, 447

Prada, F., Gutierrez, C. M., Peletier, R. F., \& McKeith, C. D. 1996, ApJ, 463, L9 
Rix, H.-W., Franx, M., Fisher, D., \& Illingworth, G. 1992, ApJ, 400, L5

Rix, H.-W. R., Kennicutt, R. C. Jr., Braun, R., \& Walterbos, R. A. M. 1995, ApJ, 438, 155

Rubin, V. 1994, AJ, 107, 173

Rubin, V., Graham, J. A., \& Kenney, J. D. P. 1992, ApJ, 394, L9

Sanchez-Blazquez, P., et al. 2006, MNRAS, 371, 703

Sarzi, M., et al. 2006, MNRAS, 366, 1151

Shalyapina, L. V., Moiseev, A. V., Yakovleva, V. A., Hagen-Thorn, V. A., \& Barsunova, O. Yu. 2004, Astron. Lett., 30, 583

Sil'chenko, O. K. 1999, AJ, 118, 186

Sil'chenko, O. K. 2005, Astron. Lett., 31, 227

Sil'chenko, O. K., \& Afanasiev, V. L. 2000, A\&A, 364, 479

Sil'chenko, O. K., \& Afanasiev, V. L. 2004, AJ, 127, 2641

Sil'chenko, O. K., \& Afanasiev, V. L. 2008, Astron. Rep., 52, 875
Sil'chenko, O. K., \& Moiseev, A. V. 2006, AJ, 131, 1336

Sil'chenko, O. K., Vlasyuk, V. V., \& Burenkov, A. N. 1997, A\&A, 326, 941

Simien, F., \& Prugniel, Ph. 2002, A\&A, 384, 371

Thakar, A. R., \& Ryden, B. S. 1996, ApJ, 461, 55

Thakar, A. R., Ryden, B. S., Jore, K. P., \& Broeils, A. H. 1997, ApJ, 479, 702

Theureau, G., et al. 1998, A\&AS, 130, 333

Tonry, J., \& Davis, M. 1979, AJ, 84, 1511

Van Albada, T. S., Kotanyi, C. G., \& Schwarzschild, M. 1982, MNRAS, 198, 303

Vergani, D., Pizzella, A., Corsini, E. M., van Driel, W., \& Buson, L. M. 2007, A\&A, 463, 883

York, D. G., et al. 2000, AJ, 120, 1579

de Zeeuw, P. T., et al. 2002, MNRAS, 329, 513 